\title{
Crecimiento y producción de Solanum gilo Raddi en sistemas de cultivo en callejones con adición de estiércol bovino
}

\author{
Growth and production of Solanum gilo Raddi in alley cropping systems with addition of cattle
} manure

Crescimento e produção do Solanum gilo Raddi em sistemas de cultivo em aleias com adição de esterco bovino

\author{
Dieny Michelly Schuertz da Silva \\ ORCID: https://orcid.org/0000-0002-8118-1784 \\ Universidade Estadual de Roraima, Brasil \\ E-mail: dienyschuertz@yahoo.com.br \\ Jandiê Araujo da Silva \\ ORCID: https://orcid.org/0000-0003-3760-3959 \\ Universidade Federal de Roraima, Brasil \\ E-mail: jandie.araujo@ufrr.br \\ Járisson Cavalcante Nunes \\ ORCID: https://orcid.org/0000-0002-5129-2934 \\ Universidade Estadual da Região Tocantina do Maranhão, Brasil \\ E-mail: jarissonagro@hotmail.com \\ Miguel A. Maffei Valero \\ ORCID: https://orcid.org/0000-0001-5234-5156 \\ Universidad de Los Andes, Venezuela \\ E-mail: migmaffei@ula.ve \\ Sonicley da Silva Maia \\ ORCID: https://orcid.org/0000-0003-0167-3218 \\ Universidade Federal de Roraima, Brasil \\ E-mail: sony_maia@hotmail.com
}

\begin{abstract}
Resumen
Los sistemas de cultivo en callejones y el uso de fertilizantes orgánicos se consideran buenas prácticas para el desarrollo de una agricultura sostenible. El objetivo de este estudio fue evaluar los efectos de la aplicación de estiércol de bovino y el uso de sistema de callejones con plantas de Gliricídia sepium sobre el crecimiento y producción de jiló en la sabana de Roraima, Brasil. El experimento en campo fue dispuesto en bloques al azar en esquema factorial $2 \times 5$, con tres repeticiones. El primer factor fue sistema de cultivo (con y sin callejones) SCC y SSC respectivamente, y el segundo factor correspondió a los niveles de materia orgánica (MOS) adicionados al suelo $(1,21 ; 2,21 ; 3,21 ; 4,21$ y 5,21\%). A los 30, 60 y 90 días después del trasplante se evaluó la altura de planta (AP), el diámetro del tallo (DT) y el número de hojas $(\mathrm{NH})$. Se recolectaron frutos semanalmente registrándose la masa promedio de frutos (MF), número de frutos por planta (NFP) y el rendimiento (RN). Las plantas del SCC tuvieron menos desarrollo en comparación con las del SSC. El aumento de MOS influyó positivamente en el desarrollo y producción del jiló. El uso de estiércol de bovino proporciona un aumento en los componentes de producción. El incremento de 5,21\% de MOS en el sistema sin callejones, brindó los mejores resultados en DT, NH, MF, NFP y RN. El cultivo con callejones no aporta una mejora en los índices de producción del jiló.
\end{abstract}

Palabras clave: Agroforestería; Cobertura de suelo; Jiló; Leguminosas arbóreas; Solanaceae.

\begin{abstract}
Alley cropping systems and the use of organic fertilizers are considered good practices for the development of sustainable agriculture. The objective of this study was to evaluate the effects of the application of bovine manure and the use of an alley system with Gliricídia sepium plants on the growth and production of jilo in the Roraima savannas, Brazil. The field experiment was arranged in random blocks in a $2 \times 5$ factorial scheme, with three repetitions. The first factor was the cultivation system (with and without alleys) CA and SA respectively, and the second factor corresponded to the levels of organic matter (MOS) added to the soil $(1.21 ; 2.21 ; 3.21 ; 4,21$, and 5.21\%). At 30, 60, and 90 days after transplantation, plant height (AP), stem diameter (DT), and number of leaves (NH) were evaluated. Fruits were collected weekly, recording the average mass of fruits (MF), number of fruits per plant (NFP), and yield (RN). The plants of the CA had less development compared to those of the SA. The increase in MOS positively influenced the development and production of the jiló. The use of bovine manure provides an increase in production components. The $5.21 \%$
\end{abstract}


increase in MOS in the system without alleys, provided the best results in DT, NH, MF, NFP, and RN. The cultivation with alleys does not provide an improvement in the jiló production indices.

Keywords: Agroforestry systems; Soil cover; Scarlet eggplant; Arboreal legumes; Solanaceae.

\begin{abstract}
Resumo
Os sistemas de cultivo em aleias e o uso de fertilizantes orgânicos são considerados boas práticas para o desenvolvimento da agricultura sustentável. O objetivo deste trabalho foi avaliar os efeitos da aplicação de esterco bovino e do uso de um sistema de aleias com plantas de Gliricídia sepium no crescimento e produção de jiló no cerrado de Roraima, Brasil. O experimento de campo foi organizado em blocos ao acaso em esquema fatorial $2 \times 5$, com três repetições. O primeiro fator foi o sistema de cultivo (com e sem aleias) CA e SA respectivamente, e o segundo fator correspondeu aos níveis de matéria orgânica (MOS) adicionados ao solo $(1,21 ; 2,21 ; 3,21 ; 4,21$ e 5,21\%). Aos 30, 60 e 90 dias após o transplante, foram avaliados a altura da planta (AP), o diâmetro do caule (DT) e o número de folhas $(\mathrm{NH})$. Os frutos foram coletados semanalmente, registrando-se a massa média dos frutos (MF), o número de frutos por planta (NFP) e a produtividade (RN). As plantas do CA tiveram menor desenvolvimento em relação às do SA. O aumento da MOS influenciou positivamente o desenvolvimento e a produção do jiló. O uso de esterco bovino proporciona aumento dos componentes da produção. O aumento de 5,21\% da MOS no sistema sem aleias proporcionou os melhores resultados em DT, NH, MF, NFP e RN. O cultivo com aleias não proporcionou melhora nos índices de produção de jiló.
\end{abstract}

Palavras-chave: Sistemas agroflorestais; Cobertura de solo; Jiló; Leguminosas arbóreas; Solanaceae.

\title{
1. Introducción
}

La preocupación por el medio ambiente y la calidad de vida ha extendido ampliamente las corrientes de la agricultura sostenible y la alimentación saludable, especialmente en relación con las hortalizas. Sobre el particular, los arreglos productivos en sistemas agroforestales y la adición de residuos orgánicos al suelo se han convertido en una opción atractiva desde un punto de vista productivo, económico y sustentable, debido a las mejoras en los atributos químicos, físicos y biológicos del suelo que proporciona la cubierta vegetal.

Desde esta perspectiva, los sistemas agroforestales son estrategias de manejo que contribuyen a incrementar la producción, un ejemplo de esto es el cultivo en callejones con Gliricidia sepium. Este sistema puede compensar los efectos negativos de la alta variabilidad de las precipitaciones en zonas con estación seca prolongada; aporta nitrógeno al suelo, el cual es fijado a través de la simbiosis de bacterias nitrificantes y las plantas de gliricidia, al mismo tiempo que contribuye a aumentar o estabilizar la disponibilidad de alimentos producidos de manera sostenible y con menor impacto ambiental (Santos, PérezMarin, \& Sarmento, 2018).

Así, la gestión sostenible de los ecosistemas agrícolas promueve una mayor productividad y mejora la calidad del suelo a través del aumento de carbono capturado, la disponibilidad de recursos hídricos y el mantenimiento de la biodiversidad, garantizando a su vez su conservación para futuras generaciones y la explotación consciente de los recursos naturales, siempre observando los principios agroecológicos, con el propósito de alcanzar la sustentabilidad de la agricultura familiar brasileña (Lal, 2009; Lucena, Silva, Silva, Silva \& Batista, 2019).

En este sentido, comprender mejor la dinámica y la función de la materia orgánica en el suelo es de relevante importancia, dado el significativo papel que desempeña en los suelos brasileños y en el manejo de estos agroecosistemas (Lucena, Silva, Silva, Silva \& Batista, 2019).

En estos sistemas, es habitual el uso de abono orgánico, lo que contribuye de forma decisiva a mejorar las características del suelo y su fertilidad, e incluso puede reducir el costo de esta práctica, ya que el abono mineral encarece la producción de los cultivos. Otra ventaja se refiere al grado de descomposición, ya que el fertilizante orgánico puede tener un efecto inmediato en la planta, o un efecto residual, a través de un proceso de descomposición más lento (Santos et al., 2006).

Al respecto, se ha demostrado que la fertilización con estiércol bovino es eficiente en la producción agrícola, constituyendo práctica común en sistemas de cultivo menos dañinos para el medio ambiente. Sin embargo, los estudios relacionados con la nutrición vegetal son fundamentales para incrementar la productividad, pues como afirma Reis (2012) las 
hortalizas reaccionan positivamente a esta fertilización, tanto en términos de productividad como de calidad del producto, siendo el estiércol bovino la fuente más utilizada por los productores.

Entre las hortalizas con potencial económico, nutricional y medicinal, destaca el jiló ( $S$. gilo), por ser un cultivo que contiene flavonoides, alcaloides y esteroides, y sus frutos tienen propiedades antioxidantes con capacidad para bajar el nivel de colesterol. Las frutas contienen aproximadamente $92,5 \%$ de agua, $1 \%$ de proteínas, $0,3 \%$ de grasas y $6 \%$ de carbohidratos, además de $34 \mathrm{mg}$ de hierro, $34 \mathrm{mg}$ de fósforo, $66 \mathrm{mg}$ de vitamina A, 0,07 $\mathrm{mg}$ de tiamina, 0,07 $\mathrm{mg}$ de riboflavina, $22 \mathrm{mg}$ de calcio y 8,6 mg de ácido ascórbico (Odetola, Iranloye, \& Akinloye, 2004).

A la fecha, la información sobre el uso de prácticas agronómicas sostenibles sobre el cultivo de jiló, que permitan el aumento y/o mantenimiento de la cantidad y calidad de materia orgánica en el suelo, es aún incipiente. Este hecho indica la necesidad de realizar investigación científica con el propósito de generar información para la comunidad académica y los productores de jiló, lo que se traduciría en mayor difusión sobre su cultivo, aumento de su productividad, contribuyendo en la mejora en las características químicas, físicas y propiedades biológicas del suelo en sus propiedades.

Por lo anteriormente planteado, se realizó una investigación de campo cuyo objetivo fue evaluar los efectos de la aplicación de estiércol de bovino y el sistema de callejones con plantas de gliricidia sobre los atributos del suelo, crecimiento y producción de jiló en la sabana de Roraima, Brasil.

\section{Metodología}

El experimento se realizó en la Escuela Agrotécnica Campus Murupu de la Universidade Federal de Roraima, municipio de Boa Vista-RR. Según la clasificación de Köppen, el clima del municipio es del tipo Aw, caracterizado como tropical lluvioso, caluroso y húmedo (Barni, Barbosa, Xaud, Xaud, \& Fearnside, 2020), con una temperatura promedio de $25^{\circ} \mathrm{C}$ y precipitaciones de 1,534 mm distribuidas irregularmente, siendo más lluvioso entre los meses de abril a septiembre (Alvares, Stape, Sentelhas, De Moraes Gonçalves, \& Sparovek, 2013); el suelo del área experimental fue clasificado como Geric Xanthic Ferralsol, de textura franco-arenosa.

El sistema de callejones estuvo conformado por plantas de gliricidia sembradas en 2013, distribuidas en marco de plantación de $7 \mathrm{~m} \mathrm{x} \mathrm{3,5} \mathrm{m;} \mathrm{las} \mathrm{plántulas} \mathrm{provinieron} \mathrm{de} \mathrm{esquejes}(25 \mathrm{~cm})$ suministrados por la Unidad Experimental de EMBRAPA-RR ubicada en el municipio de Boa Vista, Roraima. El primer año de trasplante se llevó a cabo la fertilización verde mediante siembra al voleo de crotalaria (Crotalaria juncea), guandu anão (Cajanus cajan), canavalia (Canavalia ensiformis) y mucuna preta (Mucuna pruriens). Después de este cultivo, el área estuvo en barbecho hasta 2015, y posteriormente fue cultivado maíz intercalado con las leguminosas antes mencionadas durante dos años consecutivos. El manejo del sistema consistió en la práctica de podas y la disposición de la biomasa vegetal sobre la superficie del suelo de acuerdo a lo establecido en Paula, Campello, Guerra, Santos, e de Resende (2015).

Antes de la instalación del experimento, se realizó una poda, dejando las plantas de gliricidia con 4 metros de altura, el material vegetal retirado fue depositado en el suelo aledaño a los árboles; esta práctica fue establecida ya que en correspondencia a lo planteado en Peneireiro (1999), la poda permite un mayor desarrollo de la raíz y consecuentemente mayor absorción de nutrientes del perfil del suelo. Luego, se tomó una muestra compuesta de suelo entre los 0-30 cm de profundidad en cada sistema de cultivo (con y sin callejones), las muestras fueron analizadas siguiendo la metodología propuesta por Donagema et al. (2011), sus atributos químicos y físicos son presentados en el Tabla 1.

Los tratamientos se organizaron en bloques al azar, dispuestos en un esquema factorial ( $2 \times 5)$ con arreglo en parcelas divididas repetidas tres veces; de este modo las parcelas principales estuvieron conformadas por el cultivo de jiló con y sin sistema de callejones, y las subparcelas correspondieron al incremento del contenido de materia orgánica del suelo $(1,21,2,21$, $3,21,4,21$ y $5,21 \%$ ). 
Tabla 1. Atributos químicos y físicos del suelo entre los 0-30 cm de profundidad, en un área con sistema y sin sistema de callejones (SC y SSC respectivamente), antes de la implementación del experimento.

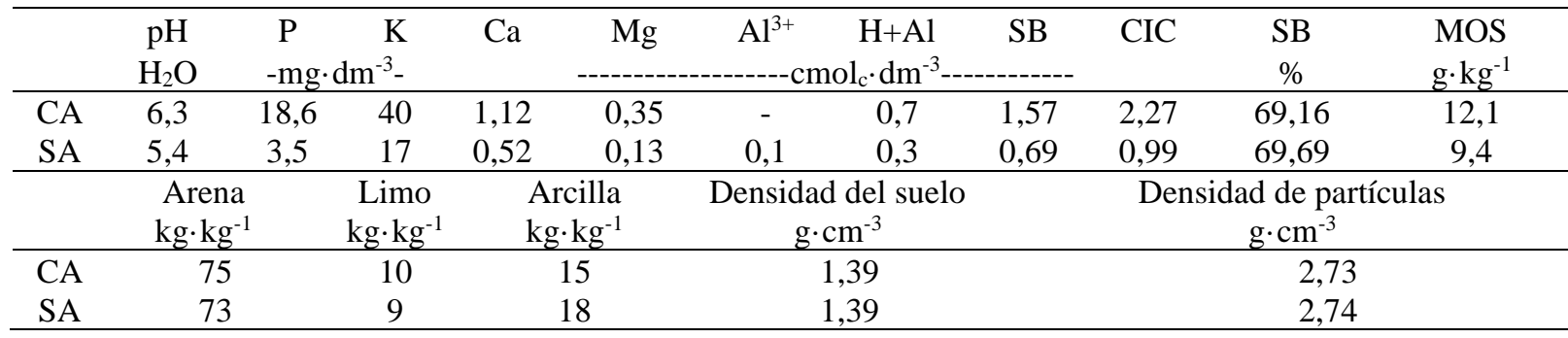

pH en agua (1:2:5); $\mathrm{Ca}, \mathrm{Mg}$ y Al$^{3+}$ : extractor $\mathrm{KCl} 1 \mathrm{~mol} \mathrm{~L}{ }^{-1}$; $\mathrm{K}$ y P: extractor Mehlich-1; $\mathrm{H}+\mathrm{Al}$ : extractor acetato de calcio. Ca: calcio; Mg: magnesio; $\mathrm{Al}^{3+}$ : aluminio; K: potasio; P: fósforo SB: suma de bases; CIC: capacidad de intercambio catiónico; SB: índice de saturación por bases; y MOS: materia orgánica del suelo. Fuente: Autores.

El crecimiento y producción del jiló fue evaluado mediante las variables altura de planta, diámetro del tallo y número de hojas, a estas variables se les hizo evaluación a los 30, 60 y 90 días después del trasplante. Para el caso de la producción fueron consideradas las variables masa promedio de frutos (MPF) expresado en gramos, número de frutos por planta (NFP) y rendimiento $(\mathrm{RN})$ en $\mathrm{t} \cdot \mathrm{ha}^{-1}$.

Para aumentar el contenido de materia orgánica a los niveles deseados se añadió estiércol bovino, ajustándose según la fórmula propuesta por Bertino et al. (2015).

$$
\mathrm{M}=\frac{(M O D-M O I) * d s * v * U}{M O E}
$$

Donde: $\mathrm{M}=$ cantidad de estiércol a aplicar $\left(\mathrm{g} \cdot \mathrm{kg}^{-1}\right) ; \mathrm{MOD}=$ Contenido de materia orgánica deseado $\left(\mathrm{g} \mathrm{kg}^{-1}\right) ; \mathrm{MOI}=$ Contenido de materia orgánica en el suelo $\left(\mathrm{g} \cdot \mathrm{kg}^{-1}\right)$; ds = densidad del suelo; $\mathrm{v}=$ volumen del agujero; $\mathrm{U}=$ Humedad del estiércol y MOE = Contenido de materia orgánica del estiércol.

El estiércol bovino se caracterizó químicamente de acuerdo a la metodología propuesta por Donagema et al. (2011) y presentó los siguientes valores: $\mathrm{pH}$ en $\mathrm{H}_{2} \mathrm{O}: 8,91 ; \mathrm{N}: 1,94 \% ; \mathrm{P}: 0,58 \mathrm{mg} \cdot \mathrm{dm}^{-3} ; \mathrm{K}: 3,20 \mathrm{mg} \cdot \mathrm{dm}^{-3} ; \mathrm{Ca}: 0,73 \mathrm{cmol} \cdot \mathrm{dm}^{-3} ; \mathrm{Mg}: 0,28$ $\mathrm{cmol} \cdot \mathrm{dm}^{-3} ; \mathrm{S}: 0,37 \mathrm{mg} \cdot \mathrm{dm}^{-3}$; Na: 0,14 $\mathrm{cmol} \cdot \mathrm{dm}^{-3} ; \mathrm{Zn}: 134 \mathrm{mg} \cdot \mathrm{dm}^{-3} ; \mathrm{Fe}: 16376 \mathrm{mg} \cdot \mathrm{dm}^{-3} ; \mathrm{Mn}: 1733 \mathrm{mg} \cdot \mathrm{dm}^{-3} ; \mathrm{Cu}: 36 \mathrm{mg} \cdot \mathrm{dm}^{-3}$; B: $15,4 \mathrm{mg} \cdot \mathrm{dm}^{-3}$; y densidad: desde $0,34 \mathrm{~g} \cdot \mathrm{cm}^{-3}$.

Cada tratamiento consistió en seis plantas de jiló espaciadas $1 \mathrm{~m}$ entre hileras y $1 \mathrm{~m}$ entre plantas, se evaluaron las dos plantas centrales, sin considerar las plantas ubicadas en los extremos (borduras).

Fueron adquiridas semillas del cultivar Redondo Morro Grande, esto por ser el de mayor disponibilidad en el mercado local y de gran aceptación por parte de los consumidores; este cultivar se caracteriza principalmente por presentar frutos redondos y con un peso entre 27 y $32 \mathrm{~g}$. Las plántulas se produjeron en un ambiente protegido, utilizando bandejas de plástico con 50 celdas y sustrato comercial (Organo Amazon ${ }^{\circledR}$ ). La siembra se realizó manualmente, colocando una semilla por celda. Las bandejas se regaron diariamente mediante regadera hasta alcanzar una altura de $10 \mathrm{~cm}$ (etapa de cuatro a cinco hojas permanentes) lo que ocurrió 40 días después de la siembra, cuando se realizó el trasplante.

El trasplante se realizó en agujeros ( 30 × 30 × $30 \mathrm{~cm})$ abiertos con 45 días de anticipación, momento en el que se realizó la corrección de acidez mediante el método de neutralización de $\mathrm{Al}^{3+}$ y elevación de los contenidos de calcio y magnesio, aplicando cal dolomítica en la cantidad de 0,96 t $\cdot$ ha $^{-1}$ (PRNT 100\%). Las dosis de estiércol bovino se aplicaron 15 días antes del trasplante y la cantidad utilizada en cada hoyo difirió según los tratamientos (Tabla 2). 
Tabla 2. Dosis de estiércol bovino aplicadas en los agujeros para el cultivo de jiló en el sistema con y sin callejones.

\begin{tabular}{ccc}
\hline Tenor de materia orgánica del suelo & \multicolumn{2}{c}{ Cantidad de estiércol aplicado $\left(\mathrm{kg} \cdot\right.$ agujero $\left.{ }^{-1}\right)$} \\
\cline { 2 - 3 }$(\%)$ & Área con callejones & 0,29 \\
\hline 1,21 & 0 & 1,34 \\
2,21 & 1,06 & 2,40 \\
3,21 & 2,12 & 3,46 \\
4,21 & 3,18 & 4,52 \\
5,21 & 4,24 & Área sallejones \\
\hline
\end{tabular}

Fuente: Autores.

Durante la conducción del ensayo se realizaron deshierbes manuales para mantenerlo libre de plantas espontáneas, fueron realizadas aplicaciones semanales alternando con caldo de azufre y extracto de Neem (Azadirachta indica) como medidas profilácticas y de control frente a la aparición de enfermedades fungosas como la mancha negra (Alternaria solani) y plagas como la chinche de encaje (Corythaica cyathicollis), trips (Thrips palmi y Frankliniella schultzei) y pulgones (Myzus persicae y Macrosiphum euphorbiae).

Las plantas fueron tutoradas para evitar volcamiento en el momento de la producción de frutos y se regaron con goteros localizados, con mangueras de $200 \mu$ de espesor y emisores espaciados $30 \mathrm{~cm}$, con una manguera de goteo instalada por cada línea de cultivo. El agua provenía de un pozo profundo y la lámina de riego se aplicó basada en la demanda del cultivo de acuerdo al estado de desarrollo del mismo; en total fueron aplicados $172 \mathrm{~mm}$ durante el ciclo de cultivo. En los días en que hubo suficiente lluvia, se suspendió el riego y se completó cuando fue necesario.

Se evaluó el desarrollo de las plantas de jiló con medidas tomadas a los 30, 60 y 90 días después del trasplante. La altura de la planta $(\mathrm{cm})$ se midió con una regla graduada colocada en la base del tallo hasta el ápice de la hoja más joven; el diámetro del tallo (mm), medido en la base del tallo de la planta, utilizando un vernier digital de precisión (0,01 mm); se contó el número de hojas por planta, considerando solo hojas activas con un tamaño igual o superior a $5 \mathrm{~cm}$.

La cosecha de ambos sistemas se inició a los 90 días después del trasplante (DDT). Los frutos fueron colectados semanalmente, totalizando siete cosechas. En cada cosecha los frutos fueron contabilizados y pesados; una vez culminado el ciclo del cultivo, fueron determinados los valores de las variables frutos por planta (frutos $\cdot$ planta $^{-1}$ ), peso medio de frutos $\left(\mathrm{g} \cdot \mathrm{fruto}^{-}\right.$ $\left.{ }^{1}\right)$ y productividad expresada en $\mathrm{t} \cdot \mathrm{ha}^{-1}$.

Al final del ciclo productivo (110 días después del trasplante) se colectaron muestras de suelo en cada tratamiento (0$30 \mathrm{~cm})$ para evaluar la fertilidad, se evaluaron los atributos químicos: $\mathrm{pH}\left(\mathrm{H}_{2} \mathrm{O}\right)$; potasio $(\mathrm{K})$; fósforo $(\mathrm{P})$; calcio $(\mathrm{Ca})$, magnesio $(\mathrm{Mg})$; aluminio $(\mathrm{Al})$ y acidez potencial $(\mathrm{H}+\mathrm{Al})$.

Los datos fueron sometidos a análisis de varianza, utilizando la prueba $\mathrm{F}$ al $5 \%$ de probabilidad, utilizando el software SISVAR $^{\odot}$ (Ferreira, 2014). Las medias del sistema de callejones se compararon mediante la prueba de Tukey y las del contenido de materia orgánica del suelo mediante regresión polinomial. Los criterios adoptados para la elección del modelo de regresión se basaron en un nivel de significancia de hasta el $5 \%$.

\section{Resultados y discusión}

\subsection{Efecto de los tratamientos sobre los atributos químicos del suelo}

Al final del ciclo del cultivo (100 DDT), a pesar de la adición de estiércol de bovino, los valores medios de MOS registrados para los tratamientos CA y SA fueron de $8,7 \mathrm{~g} \cdot \mathrm{kg}^{-1}$ y $9,8 \mathrm{~g} \cdot \mathrm{kg}^{-1}$ (Tabla 3), lo que indica que fueron inferiores a los constatados al inicio del experimento. Adicionalmente se observó que hubo acidificación del suelo en ambos sistemas de cultivo, así como el mantenimiento durante la durante el experimento de las bases intercambiables y una disminución de la materia orgánica del suelo. 
Tabla 3. Atributos químicos del suelo entre $10 \mathrm{~s} 0-30 \mathrm{~cm}$ de profundidad, en un área con sistema y sin sistema de callejones (SC y SSC respectivamente), después de la implementación del experimento.

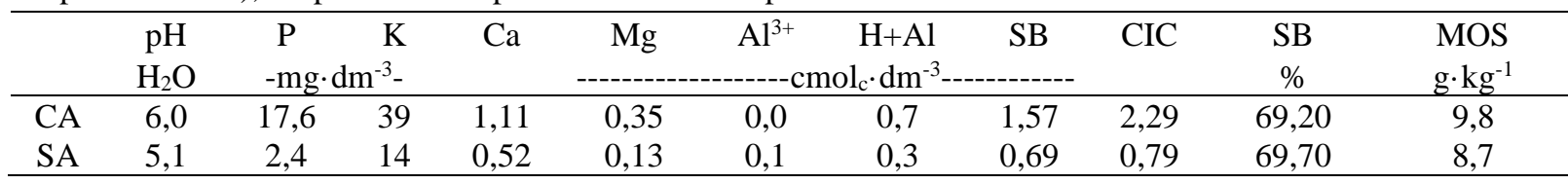

pH en agua (1:2:5); Ca, $\mathrm{Mg} \mathrm{y} \mathrm{Al}^{3+}$ : extractor $\mathrm{KCl} 1 \mathrm{~mol} \mathrm{~L}^{-1} ; \mathrm{K}^{+}$y P: extractor Mehlich-1; $\mathrm{H}+\mathrm{Al}$ : extractor acetato de calcio. Ca: calcio; $\mathrm{Mg}$ : magnesio; $\mathrm{Al}^{3+}$ : aluminio; $\mathrm{K}$ : potasio; $\mathrm{P}$ : fósforo $\mathrm{SB}$ : suma de bases; $\mathrm{CIC}$ : capacidad de intercambio catiónico; SB: índice de saturación por bases; y MOS: materia orgánica del suelo. Fuente: Autores.

Si se comparan los valores de MOS en ambos sistemas, determinados antes (Tabla 1) y después de concluido el experimento (Tabla 3), se constata que se produjo una disminución de 10,4\% y 19,1\% pasa CA y SA respectivamente; no obstante, el los tratamientos CA los tenores de MOS fueron 12,6\% superiores a los tratamientos en el sistema SA. Este resultado posiblemente esté relacionado a la práctica de la adición de biomasa vegetal sobre el suelo, resultado de la poda de plantas de gliricidia.

A pesar de la adición de estiércol de bovino se registraron bajos tenores de MOS al final del experimento, esta situación pudo estar influenciada de la acción de la temperatura y humedad del suelo del sitio del experimento, teniendo en cuenta que en las zonas de sabana como las de Roraima, la tasa de pérdida de carbono soy muy elevadas (Melo, Evald, Roberto, \& Rocha, 2017; Souza, Melo, Araújo \& Araújo, 2019).

Adicionalmente, de acuerdo con datos oficiales del Instituto Nacional de Meteorología, durante el período experimental de noviembre de 2018 a mayo de 2019, en el municipio de Boa Vista la precipitación registrada fue de 537,2 mm y temperatura media del período de $28,5^{\circ} \mathrm{C}$ (INMET, 2020).

En este aspecto, estudios muestran que más del 50\% de la materia orgánica del estiércol de ganado vacuno, caprino y ovino desaparece en un período de aproximadamente 20 semanas y que la mineralización varía según los compuestos del estiércol, como la lignina y los polifenoles (Esse, Buerkert, Hiernaux, \& Assa, 2001). Por tanto, pueden producirse pérdidas de materia orgánica del suelo por lixiviación, erosión y descomposición. Diversos estudios demuestran que muchas variables pueden influir en este atributo en el suelo. A escala regional, las variables climáticas, como la temperatura y las precipitaciones son importantes; a nivel local, se destacan las propiedades del suelo, como la densidad y la fertilidad natural (Assad et al., 2013).

En este sentido, si bien el sistema con callejones ha influido en la reducción de materia orgánica, con el tiempo promueve mayores beneficios de este atributo al suelo, ya que las plantas de gliricidia son capaces de fijar cantidades considerables de $\mathrm{N}$ del aire al suelo, además a producir considerable cantidad de biomasa ((Alves, Abboud, Ribeiro, \& Almeida, 2004), otra ventaja es que puede brindar sombra y proteger contra los vientos, creando un microclima favorable. Por esta razón, el uso de esta planta, además de representar ahorro en fertilizantes principalmente nitrogenados, contribuye al manejo ecológico, que es fundamental para la producción orgánica (Espindola et al., 2006).

Es importante agregar que el uso de G. sepium puede considerarse una forma viable de mitigar los impactos de la agricultura, aportando sostenibilidad al manejo de suelos agrícolas, ayudando a agilizar el ciclo de nutrientes, contribuyendo al ahorro de agua, protección contra plagas y enfermedades, y en el control de la erosión laminar, produciendo polen y néctar para enemigos naturales, enriqueciendo la estructura del suelo al agregar materia orgánica y actividad de los sistemas radiculares de estas plantas, además de reducir la infestación por plantas espontáneas (Hilton et al., 2016; Leal et al., 2021).

\subsection{Altura de planta de jiló (AP), diámetro de tallo (DT) y número de hojas (NH)}

De acuerdo al resumen del análisis de varianza para AP, DC y NF, se puede observar que hubo efecto de interacción para todas las variables estudiadas en función del contenido de materia orgánica del suelo $\times$ sistema de callejones (Tabla 4). 
Tabla 4. Resumen del ANOVA para la altura de la planta de jiló (AP), el diámetro del tallo (DT) y el número de hojas (NH), del contenido de materia orgánica del suelo y el sistema de callejones en EAgro, Boa Vista-RR.

\begin{tabular}{lcccc}
\hline \multicolumn{1}{c}{ FV } & GL & AP & DT & NH \\
\hline Bloque & 2 & $7,54^{\text {ns }}$ & $1,31^{* *}$ & $2,56^{*}$ \\
Callejones (A) & 1 & $60,69^{*}$ & $80,27^{*}$ & $228,70^{* *}$ \\
Error A & 2 & 1,08 & 0,052 & 0,38 \\
\hline Parcelas & 5 & - & - & - \\
\hline Dosis (D) & 4 & $101,43^{*}$ & $8,20^{*}$ & 22,83 \\
AxD & 4 & $1,55^{*}$ & $0,14^{*}$ & 1,76 \\
Error B & 16 & 51,91 & 12,63 & - \\
Total (Subparcelas) & 29 & - & - & 4,98 \\
CV 1 (\%) & & 8,32 & 2,40 & 4,90 \\
CV 2 (\%) & & 9,94 & 4,02 & \\
\hline
\end{tabular}

Fuente: Autores.

Según el análisis de regresión, los promedios de altura de planta y el diámetro del tallo se ajustaron al modelo lineal (Figuras 1 y 2) y el número de hojas se ajustó al modelo de regresión cuadrático (Figura 3) estos son graficados en función del contenido de materia orgánica del suelo.

Figura 1. Altura de las plantas de jiló en el sistema con callejones (A) y sin callejones (B) a los 30, 60 y 90 días después del trasplante en función del contenido de materia orgánica del suelo (MOS).

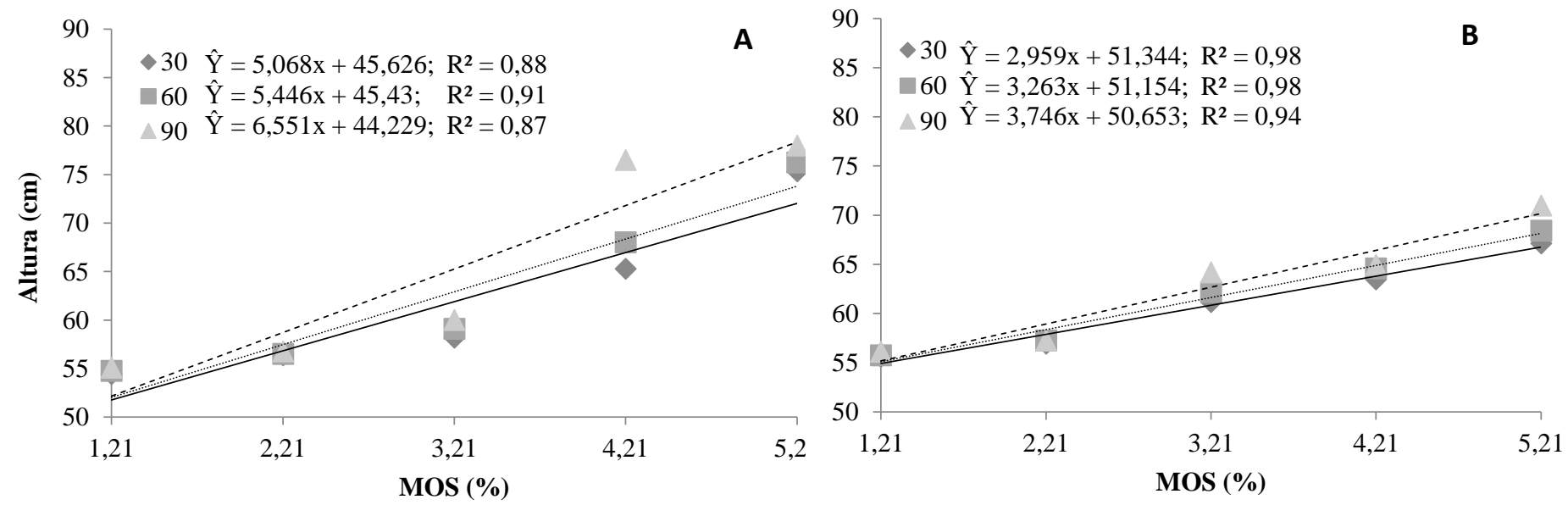

Fuente: Autores.

Las plantas de jiló del sistema con callejones tuvieron mayor desarrollo en altura (Figura 1A), en comparación con el sistema convencional (sin callejones) (Figura 1B). También se verificó mayor AP según el incremento de materia orgánica en el suelo, donde el mejor porcentaje fue de $5,21 \%$, que corresponde a 42,4 $\mathrm{t} \cdot \mathrm{ha}^{-1}$ de estiércol bovino. Resultados contrastantes fueron verificados por Barros (2018) en un experimento con biofertilizante líquido sobre el comportamiento agronómico del cultivar jiló $c v$. Tinguá en producción orgánica, en el cual se encontró una altura promedio de planta de 63,54 cm a los 40 días después del trasplante.

Este resultado probablemente pueda estar asociado con el principio de exclusión competitiva citado por Gause (1934), porque cuando la competencia entre dos especies es lo suficientemente fuerte, una de ellas perecerá. Así, parece que el manejo de las plantas de gliricidia, en el sistema de callejones puede no haber sido suficiente para que las plantas de jiló aprovecharan los recursos disponibles en el medio de estos callejones, principalmente luz solar. 
Los estudios de los consorcios de cultivo de árboles se centran principalmente en la competencia indirecta por la explotación de recursos compartidos. En este sentido, la competencia por la radiación solar es, en la superficie, más relevante entre árboles y cultivos. Una alternativa para paliar esta competencia es a través de la poda, ya que además de suavizar la sombra de los cultivos, agrega biomasa para la cobertura del suelo y abono verde (Bernardes, Pinto, \& Righi, 2009).

Además, según Taiz, Zeiger, Moller, \& Murphy (2017) para una "planta solar", existe un claro valor adaptativo en la asignación de sus recursos para un crecimiento más rápido cuando está bajo la sombra de otra planta. De esta manera, puede aumentar sus posibilidades de crecer por encima del dosel y adquirir una mayor porción de radiación fotosintéticamente activa sin filtrar.

El diámetro del tallo aumentó a lo largo de las evaluaciones de desarrollo de las plantas de jiló, de acuerdo a la adición de materia orgánica al suelo. En el sistema con callejones (Figura 2A) se registraron las medias más bajas en comparación con el sistema sin callejones (Figura 2B).

Figura 2. Diámetro del tallo de plantas de jiló en el sistema con callejones (A) y sin callejones (B), a los 30, 60 y 90 días después del trasplante en función del contenido de materia orgánica del suelo (MOS).
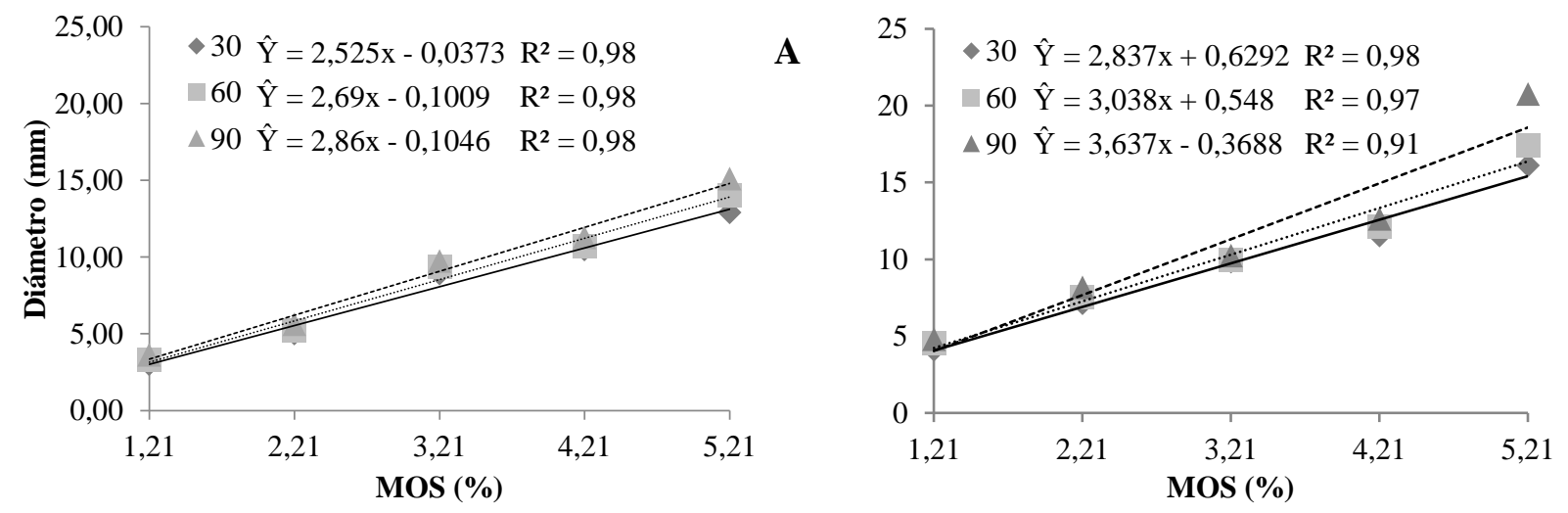

Fuente: Autores.

Este resultado puede estar asociado con las mejores condiciones de luz en el área que se cultivó sin el sistema de callejones. Además de esto, la aplicación de estiércol de vacuno al suelo puede haber contribuido al aumento de la disponibilidad de nutrientes, cumpliendo con los requerimientos nutricionales del cultivo y proporcionando un mejor aprovechamiento de los nutrientes, lo que se refleja en mayores valores de diámetro del tallo (Santos et al., 2006). Esta variable se asocia a un desarrollo más acentuado de la parte aérea y, en particular, del sistema radicular, beneficiando la supervivencia y desarrollo de la planta en campo (Santos et al. 2017).

Para el número de hojas por planta, el tratamiento con callejones mostró los promedios más altos, con valores crecientes a medida que aumentaba la edad de las plantas y se agregaba materia orgánica al suelo (Figura 3).

El mejor resultado de esta variable puede estar relacionado con la naturaleza orgánica del material que constituye el estiércol bovino, que presenta buena disponibilidad de nutrientes (Góes, Mendonça, de Medeiros, da Silva, \& de Medeiros, 2010). En este sentido, Lima, De Souza, De Oliveira, e Martinez, 2013 (2013) también encontraron respuestas positivas en el número de hojas, frutos y flores de plantas de tomate fertilizadas con estiércol de ganado bovino. 
Figura 3. Número de hojas de plantas de jiló, en el sistema con callejones (A) y sin callejones (B), a los 30, 60 y 90 días después del trasplante en función del contenido de materia orgánica del suelo (MOS).
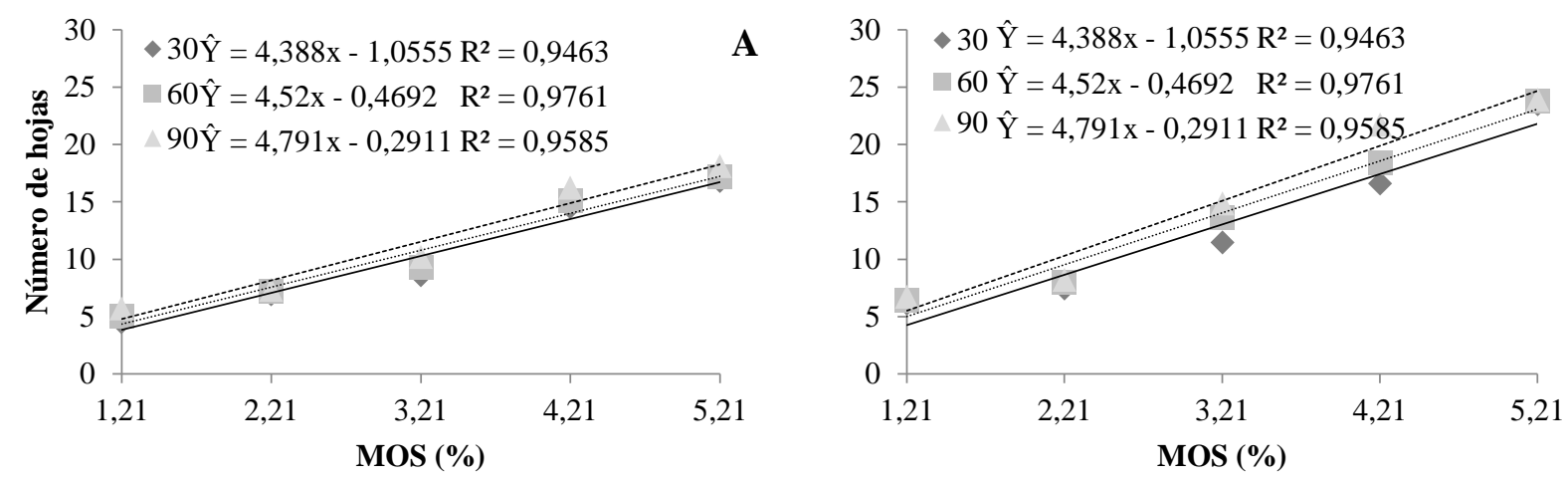

Fuente: Autores.

\subsection{Masa promedio de frutos (MPF), número de frutos por planta (NFP) y rendimiento (RN)}

La masa media de frutos tuvo un crecimiento lineal a medida que aumentaba la materia orgánica del suelo y se observó un efecto más expresivo en el sistema sin callejones (Figura 4).

Figura 4. Masa promedio de los frutos de jiló, en el sistema con callejones y sin callejones en función del contenido de materia orgánica del suelo.

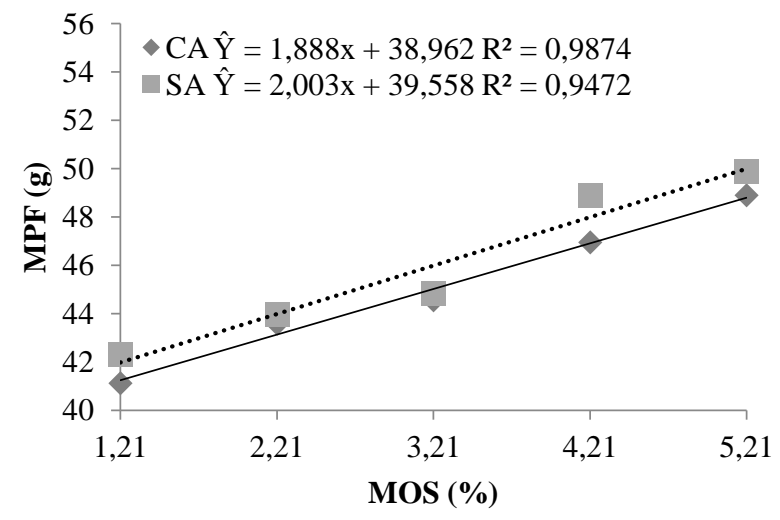

Fuente: Autores.

Con respecto a la fisiología del crecimiento y maduración de frutos de jiló, Mendes (2013) encontraron un aumento significativo en la masa de frutos entre 60 y 90 días después del inicio de la producción, con ganancias de hasta 7 gramos por fruto por día. La tasa máxima de ganancia diaria ocurrió 28 días después de la apertura de la flor, cuando los frutos mostraron incrementos de 1,07 gramos en masa seca por día. Es así que las plantas productivas extraen más nutrientes del suelo, según estudios realizados por Silva, Neto, Carneiro, e Paludo (2011) los frutos son las principales vías de drenaje de fotoasimilados en la fase reproductiva de las plantas.

Además de la masa de frutos, el incremento de materia orgánica en el suelo promovió ganancias de frutos por planta (Figura 5A) y consecuentemente mayor rendimiento (Figura 5B), en el sistema sin callejones se presentaron los promedios más altos, con incremento en el rendimiento de las plantas de 9,16 t (48,23\%) cuando se aplicó la dosis de 5,21\%. En el sistema con el callejón, hubo un aumento del 36,88\% en el rendimiento. 
Figura 5. Número de frutos. planta $^{-1}$ (A) y rendimiento de jiló (B) en el sistema con callejones y sin callejones en función del contenido de materia orgánica del suelo.
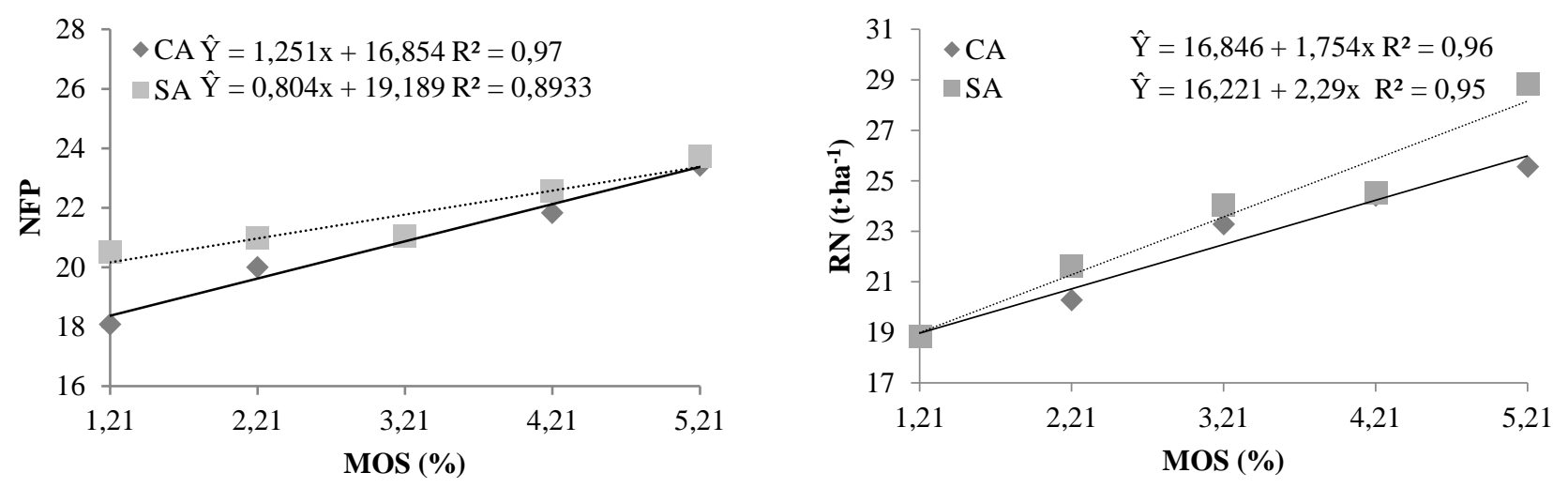

Fuente: Autores.

Resultados similares fueron reportados por Blat, Branco, e Trani (2014), estos autores estudiaron siete cultivares de jiló bajo sistemas de cultivo convencional y labranza cero, donde el cultivar Redondo Morro Grande presentó un promedio de 20,57 frutos. planta $^{-1}$, independientemente del sistema de cultivo. Por otro lado, para el cultivo de tomate, Silva et al. (2019) evaluaron la producción en función de la fertilización orgánica (estiércol bovino) y obtuvieron a la dosis de $10 \mathrm{~kg} \cdot \mathrm{ha}^{-1} 5,8$ frutos en promedio por planta, ya con la más alta la dosis de $20 \mathrm{~kg} \cdot h a^{-1}$ de estiércol, reportan una producción de 20,5 frutos por planta.

Cuando aumenta el número de frutos por planta aumenta la demanda de fotoasimilados por parte de los frutos, creando una fuerte competencia por asimilados (Shirahige, de Melo, Purquerio, Carvalho, \& de Melo, 2010). Los resultados obtenidos en el sistema sin callejones pueden justificarse por esta demanda de fotoasimilados, en los que la planta tuvo mayor actividad fotosintética necesaria para la producción de frutos.

\section{Consideraciones Finales}

El uso de estiércol de bovino proporciona un aumento en los componentes de producción analizados en el cultivo de jiló.

El incremento de materia orgánica, en el sistema sin callejones, brinda los mejores resultados en diámetro de tallo, número de hojas, masa promedio de frutos, número de frutos por planta y rendimiento de jiló;

El sistema de callejones no aporta una mejora en los índices productivos en el cultivo de jiló. Sin embargo, a largo plazo, puede contribuir en el aumento de materia orgánica en el suelo.

Recomendamos nuevos estudios en esta línea de investigación con otras especies de solanáceas como ajíes picantes y dulces tales como: Capsicum annuum (pimenta doce); C. frutescens (malagueta); C. baccatum pendulum (dedo de moça e cambuci); C. chinense (pimenta de cheiro, biquinho, murupi) y C. baccatum. praetermissum (cumari), que son de interés para los pequeños productores de las zonas de sabana en Roraima.

\section{Agradecimientos}

Los autores desean agradecer la colaboración de la Universidade Estadual de Roraima, curso de pós-graduação em Agroecologia; también al personal de campo y laboratorio de la Escuela Agrotécnica Campus Murupu de la Universidade Federal de Roraima por el apoyo institucional en la realización de esta investigación. 


\section{Referencias}

Alvares, C. A., Stape, J. L., Sentelhas, P. C., De Moraes Gonçalves, J. L., \& Sparovek, G. (2013). Köppen’s climate classification map for Brazil. Meteorologische Zeitschrift, 22(6), 711-728. https://doi.org/10.1127/0941-2948/2013/0507

Alves, S. M. C., Abboud, A. C. de S., Ribeiro, R. de L. D., \& Almeida, D. L. de. (2004). Balanço do nitrogênio e fósforo em solo com cultivo orgânico de hortaliças após a incorporação de biomassa de guandu. Pesquisa Agropecuária Brasileira, 39(11), 1111-1117.

Assad, E. D., Pinto, H. S., Martins, S. C., Groppo, J. D., Salgado, P. R., Evangelista, B., \& Martinelli, L. A. (2013). Changes in soil carbon stocks in Brazil due to land use: Paired site comparisons and a regional pasture soil survey. Biogeosciences, 10(10), 6141-6160. https://doi.org/10.5194/bg-10-6141-2013

Barni, P. E., Barbosa, R. I., Xaud, H. A. M., Xaud, M. R., \& Fearnside, P. M. (2020). Precipitação no extremo norte da Amazônia: distribuição espacial no estado de Roraima, Brasil. Sociedade \& Natureza, 32, 439-456. https://doi.org/10.14393/sn-v32-2020-52769

Barros, G. C. (2018). Utilização de biofertilizante líquido no desempenho agronômico de jiló (Solanum gilo Raddi) sob produção orgânica. Anápolis: Centro Universitário de Anápolis -UniEvangélica. 23p.

Bernardes, M. S., Pinto, L. F. G., \& Righi, C. A. (2009). Interações biofísicas em sistemas agroflorestais. Alternativa Agroflorestal Na Amazônia Em Transformação, 423-446.

Bertino, A. M. P., Evandro, F. de M., Francisco, V. D. S. S., Lourival, F. C., Nubia, M. F., Emanoela, P. de P., \& Antonio, M. P. B. (2015). Growth and gas exchange of okra under irrigation, organic fertilization and cover of soil. African Journal of Agricultural Research, 10(40), 3832-3839. https://doi.org/10.5897/ajar2015.9844

Blat, S. F., Scaloppi, E. A., Branco, R. B., \& Trani, P. E. (2014). Desempenho de cultivares de jiló em sistemas de plantio convencional e direto sob palhada de milheto. Horticultura Brasileira, 31(2), S1594 - S1601.

Donagema, G. K., de Campos, D. B., Calderano, S. B., Teixeira, W. G., \& Viana, J. H. (2011). Manual de métodos de análise de solo. (2a ed.), Rev. Embrapa Solos-Documentos (INFOTECA-E). 230p.

Espindola, J. A. A., Marinho Guerra, J. G., Perin, A., Teixeira, M. G., De Almeida, D. L., Urquiaga, S., \& Briançon Busquet, R. N. (2006). Banana plants intercropped with perennial herbaceous legumes used as living mulches. Pesquisa Agropecuaria Brasileira, 41(3), 415-420. https://doi.org/10.1590/s0100$204 \times 2006000300007$

Esse, P. C., Buerkert, A., Hiernaux, P., \& Assa, A. (2001). Decomposition of and nutrient release from ruminant manure on acid sandy soils in the Sahelian zone of Niger, West Africa. Agriculture, Ecosystems and Environment, 83(1-2), 55-63.

Ferreira, D. F. (2014). Sisvar: a Guide for its Bootstrap procedures in multiple comparisons. Ciência e agrotecnologia, 38, 109-112.

Gause G. F. (1934). The struggle for existence. Baltimore: Willians \& Wilkins, 163 p.

Góes de, G. B., Mendonça, V., de Medeiros, P. V. Q., da Silva Tosta, M., \& de Medeiros Mendonça, L. F. (2010). Diferentes substratos na produção de mudas de mamoeiro em bandejas. Revista Verde de Agroecologia e Desenvolvimento Sustentável, 5(1), 26.

Hilton Wolschick, N., Tondello Barbosa, F., Bertol, I., Fiorentin dos Santos, K., de Souza Werner, R., \& Bagio, B. (2016). Cobertura do solo, produção de biomassa e acúmulo de nutrientes por plantas de cobertura. Revista de Ciências Agroveterinárias, 15(2), 134-143. https://doi.org/10.5965/223811711522016134

Instituto Nacional de Metereologia - INMET. (2021). Banco de dados meteorológicos para ensino e pesquisa. INMET, Brasil. https://bdmep.inmet.gov.br/

Lal, R. (2009). Laws of sustainable soil management. Sustainable Agriculture, 9-12.

Leal, M. L. de A., Chaves, J. da S., Silva, J. A. da, Silva, L. S. da, Soares, R. B., Nascimento, J. P. S. do, \& Brito Neto, A. F. de. (2021). Efeito dos sistemas de manejo e do uso do solo na população de microrganismos do solo. Research, Society and Development, 10(9), e21910917966. https://doi.org/10.33448/rsdv10i9.17966

Lima Souza Maia, J. T., Clemente, J. M., De Souza, N. H., De Oliveira Silva, J., \& Prieto Martinez, H. E. (2013). Adubação orgânica em tomateiros do grupo cereja. Biotemas, 26(1), 37-44. https://doi.org/10.5007/2175-7925.2013v26n1p37

Lucena, W. B, Silva, A. L., Silva, J. L. F., Silva, H. I., \& Batista, M. C. (2019). Matéria orgânica e biologia do solo. IV Congresso Internacional das Ciências Agrárias - COINTER - PDVAGRO. 17p. https://doi.org/10.31692/2526-7701.IVCOINTERPDVAgro.2019.0137

Melo, V. F., Evald, A., Roberto, P., \& Rocha, R. (2017). Chemical and biological quality of the soil in different systems of use in the savanna environment = Qualidade química e biológica do solo em diferentes sistemas de uso em ambiente de savana. Revista Agro@Mbiente On-Line, 11(2), 101-110. https://doi.org/10.18227/1982-8470ragro.v11i2.3850

Mendes T. D. C. (2013). Growth and physiology of ripening in fruits of jilo (Solanum gilo). Tese (Doutorado em Controle da maturação e senescência em órgãos perecíveis, Fisiologia molecular de plantas superiores) - Universidade Federal de Viçosa, Viçosa, 86p.

Odetola, A. A., Iranloye, Y. O., \& Akinloye, O. (2004). Hypolipidaemic Potentials of Solanum melongena and Solanum gilo on Hypercholesterolemic Rabbits. Pakistan Journal of Nutrition, 3(3), 180-187. https://doi.org/10.3923/pjn.2004.180.187

Paula de, P. D., Campello, E. F. C., Guerra, J. G. M., Santos, G. de A., \& de Resende, A. S. (2015). Decomposição das podas das leguminosas arbóreas Gliricidia sepium e Acacia angustissima em um sistema agroflorestal. Ciencia Florestal, 25(3), 791-800. https://doi.org/10.5902/1980509819696 
Research, Society and Development, v. 10, n. 15, e470101522729, 2021

(CC BY 4.0) | ISSN 2525-3409 | DOI: http://dx.doi.org/10.33448/rsd-v10i15.22729

Peneireiro, F. M. (1999). Sistemas agroflorestais dirigidos pela sucessão natural: um estudo de caso. Dissertação apresentada à Escola Superior de Agricultura "Luiz de Queiroz", para obtenção do título de Mestre em Ciências, Área de Concentração: Ciências Florestais. Universidade de São Paulo: São Paulo, Brasil. 149 p.

Reis Filgueira, F. A. (2012). Novo manual de olericultura: agrotecnologia moderna na produção e comercialização de hortaliças. (3a ed.), Universidade Federal de Viçosa. 421p.

Santos, A. F. dos, Pérez-Marin, A. M., \& Sarmento, M. I. de A. (2018). Produtividade da palma forrageira em aleias com Gliricídia sepium sob adubação orgânica em diferentes espaçamentos no Semiárido. Revista Verde de Agroecologia e Desenvolvimento Sustentável, $13(3), 276$. https://doi.org/10.18378/rvads.v13i3.5740

Santos, J. F. dos, Oliveira, A. P. de, Alves, A. U., Brito, C. H. de, Dornelas, C. S., \& Nóbrega, J. P. (2006). Produção de batata-doce adubada com esterco bovino em solo com baixo teor de matéria orgânica. Horticultura Brasileira, 24(1), 103-106. https://doi.org/10.1590/s0102-05362006000100021

Santos, S. T. dos, De Oliveira, F. D. A., De Medeiros Costa, J. P. B., Souza Neta, M. L. de, Alves, R. D. C., \& Costa, L. P. (2017). Qualidade de mudas de cultivares de tomateiro em função de soluções nutritivas de concentrações crescentes. Revista Agro@Mbiente On-Line, 10(4), 326. https://doi.org/10.18227/1982-8470ragro.v10i4.3096

Shirahige, F. H., de Melo, A. M., Purquerio, L. F. V., Carvalho, C. R. L., \& de Melo, P. C. T. (2010). Produtividade e qualidade de tomates Santa Cruz e Italiano em função do raleio de frutos. Horticultura Brasileira, 28, 292-298.

Silva da, R. V., da Silva, V. L., de Souza, M. E., da Silva, E. C., Barbosa, R. B., Silva, M. G. M., \& Fagudes, M. K. (2019). Produção de tomateiro santa cruz em função da adubação orgânica, em nova Xavantina-MT. Campo Digital, 14(1).

Silva da, T. O., Neto, A. E. F., Carneiro, L. F., \& Paludo, V. (2011). Plantas de cobertura submetidas a diferentes fontes de fósforo em solos distintos. Semina:Ciencias Agrarias, 32(4), 1315-1326. https://doi.org/10.5433/1679-0359.2011v32n4p1315

Souza de, F. G. D., Melo, V. F., Araújo, W. F., \& Araújo, T. H. D. C. (2019). Losses of soil, water, organic carbon and nutrients caused by water erosion in different crops and natural savannah in the northern Amazon. Revista Ambiente \& Agua, 14. https://doi.org/10.4136/ambi-agua.2126

Taiz, L., Zeiger, E., Moller, I. M., \& Murphy, A. (2017). Fisiologia e Desenvolvimento Vegetal. Artmed, (6a ed.), Brasil. 888p. 